\title{
The determinants of arithmetic skills in young children: some observations
}

\author{
Haskell, Simon H
}

\begin{abstract}
The cause or causes of arithmetic difficulties in young children are manifold. The condition has its origins in a set of complex disorders and may be due to genetic factors, developmental delays, experiential limitations, language problems, and perceptual, motor, memory and other cognitive weaknesses. Other factors which may cause the disorder are inappropriate and ineffectual instructional practices. Anxiety and unfavourable attitudes towards the subject may influence mastery of arithmetic attainment in young children
\end{abstract}

DOI: https://doi.org/10.1007/s007870070011

Posted at the Zurich Open Repository and Archive, University of Zurich ZORA URL: https://doi.org/10.5167/uzh-156309

Journal Article

Published Version

Originally published at:

Haskell, Simon H (2000). The determinants of arithmetic skills in young children: some observations. European Child Adolescent Psychiatry, 9(Suppl 2):S77-S86.

DOI: https://doi.org/10.1007/s007870070011 
S. H. Haskell

Simon H. Haskell ( $ه)$

Institute for Special Education

University of Zurich

Hirschengraben 48

8001 Zurich, Switzerland

\section{The determinants of arithmetic skills in young children: some observations}

\begin{abstract}
The cause or causes of arithmetic difficulties in young children are manifold. The condition has its origins in a set of complex disorders and may be due to genetic factors, developmental delays, experiential limitations, language problems, and perceptual, motor, memory and other cognitive weak-

nesses. Other factors which may cause
\end{abstract}

the disorder are inappropriate and ineffectual instructional practices. Anxiety and unfavourable attitudes towards the subject may influence mastery of arithmetic attainment in young children.

Key words Arithmetic skills difficulties - complex disorders

\section{Introduction}

Interest in the arithmetical development and ability of young children dates back several decades. A review of the literature on the subject reveals a growing interest and an increase in research on this topic since the 1960s and early 1970s. However, the list does not match in quantity the large number of research studies on reading and reading disorders.

Knowledge about the development of arithmetical skills in young children is derived from several cognate areas, including neurology, psychology, pedagogy and sociology. It is not implied that this review provides an exhaustive or complete coverage of areas related to the subject. Issues related to definition, terminology, and prevalence are examined. The neurobiological and associated medical determinants are explored as well as psychological factors. Cultural, teaching and instructional influences on arithmetical achievement in young children are discussed.

\section{Definitions}

Confusion generally arises when such terms as mathematics, arithmetic, mathematical disabilities (MD), specific arithmetic difficulties (SAD) and developmental dyscalculia (DC) are used interchangeably. There is also a requirement to distinguish between arithmetic and mathematics in studies related to the development of arithmetic abilities in young children. Earlier definitions of mathematics embraced arithmetic geometry and algebra. Today the term incorporates other specialisations, for example, number theory, calculus, analytical geometry, set theory and a variety of other domains.

Mathematics itself invokes high-level cognitive skills involving the manipulation of mathematical operations and a conceptual grasp of mathematical problem solving. Mathematics in effect is a study of consistent formal structures, based on particular rules derived from logically compatible sets of axioms. 
Arithmetic on the other hand, requires an understanding of number facts, counting, ordering numbers serially, a mastery of number quantities, the reading and manipulation of symbols, and a knowledge of the rules governing the four basic operations of addition, subtraction, multiplication and division. In arithmetic, a number of discrete operations must be carried out in their correct sequence. For instance, addition involves the organisation of digits in a strict conventional order. Symbols are employed to indicate the nature of the operations to be carried out, for example the sign (+) is an order to add. In all these operations unambiguous rules govern the spatial arrangements of the notation system.

Mathematical disabilities (MD) as a term generally refers to children with markedly poor skills at employing "computational procedures to solve arithmetic problems and difficulties in the representation and retrieval of basic arithmetic facts from long term semantic memory" (26).

Specific arithmetic difficulties (SAD) according to Lewis et al. (52) indicates a difficulty in arithmetic alone for a child of normal intelligence.

Developmental dyscalculia (DC) according to the American Psychiatric Association (1) is a cognitive disorder of genetic origin which affects the acquisition of simple arithmetical skills in children of normal intelligence. The Diagnostic and Statistical Manual-IV edition (DSM-IV) (2) refers to the phenomenon as arithmetic performance substantially below that expected for age, intelligence and education. Kosc (51) has argued that it is a structural disorder arising from insult to a particular region of the brain which controls mathematical competence. It does not, though, affect the general intelligence of the child. Other workers have described DC as the discrepancy of at least two standard deviations of a standardised IQ and arithmetic scores.

Some stress that in the absence of diagnostic neurological signs it is difficult to establish whether or not a child suffers from a primary learning disorder which affects arithmetic skills, or experiences specific arithmetic problems exclusively. Clinicians generally disagree about the aetiology of arithmetic disorders. There remain too many outstanding questions to be answered before one is able to identify the major underlying causes of the disorder. Neuro-psychological investigations fail to offer unequivocal testimony that focal lesions result in specific arithmetic deficits. Focal cerebral injury may have diffuse sequelae and it is argued that test measures fail to detect weaknesses in such aspects of arithmetic operations as retrieval of facts from long-term memory and number encoding. Hecaen \& Albert (43) have reminded us that "cerebral localisation of function is an extremely complex phenomenon" and that the brain is not a "mosaic of clearly delimited centers, each of which is responsible for a precise psychological function". Nevertheless authors of a number of other studies claim that specific neurological insults to the brain target arithmetic and mathematical abilities in a variety of ways (see 26). Lewis and his associates (52) have suggested that arithmetic skills are impaired when selective injury to the parts of the brain which serve calculation are implicated.

\section{Prevalence rates}

Prevalence rates for arithmetic disorders or developmental dyscalculia vary between 1.6 to $6.5 \%$ in the general population in studies undertaken in the USA, England, Germany, Switzerland and Israel $(6,37,52,80)$. According to Gross-Tsur et al. (39) the higher figures in some surveys may have been inflated by the inclusion of slow learning children. Lewis, Hitch, and Walker (52) have identified three sub-types of scholastic underachievers. The first sub-group consists of children of normal intelligence who encounter specific arithmetic problems alone (SAD). The next group embraces children of normal intelligence with reading difficulties (SRD). The third sub-group comprises children of normal intelligence with arithmetic and reading problems (ARD).

A German study (50) reported a prevalence figure of $4.4 \%$ of achievement difficulties in 546 children tested on the General School Achievement test. A larger number of girls than boys showed weaknesses in mathematics in this survey. In Switzerland, von Aster et al. (83) found that almost 5\% of 279 second and fourth grade Zurich schoolchildren showed weaknesses in number processing and calculation.

Lewis et al. (52) in an epidemiological study examined the arithmetic attainments of 1206 British boys and girls between the ages of 9-10 years. They administered the Young's Group Mathematics test which includes items on addition, subtraction, multiplication and division. The authors employed cut off, rather than aptitude scores in order to establish arithmetic disabilities. The results revealed that $1.3 \%$ of the sample showed specific arithmetic difficulties (SAD) and that boys and girls were affected in equal numbers.

Gross-Tsur et al. (39) in a cohort of 3029 fourth grade Israeli children identified 143 eleven year old children exhibiting developmental dyscalculia. Their study revealed a prevalence rate of $4.1 \%$ and both sexes were similarly affected.

\section{Neurobiological aspects}

\section{Genetic influences}

It is hypothesised that arithmetic disorders may have a genetic link or have familial predispositions. Clues relating to the neurological and biological substrates of brain involvement are indicated in MRI findings. Nevertheless giftedness or weak- 
nesses in arithmetic are influenced by promising or deprived environmental circumstances. Impoverished environmental conditions may further exacerbate existing lesions to neural structures.

Neurologists and clinical psychologists persist in questioning the plausibility of various neurological explanations of arithmetic disorders in young children. Ginsburg (33) warns against the view that neurological disorders necessarily give rise to mathematical disabilities. He claims that the evidence linking disordered processes in numerical skills to specific brain damage is weak. Gordon (36) however maintains that "both cerebral hemispheres appear to contribute to mathematical skills in different ways and it may be that only very specific deficits in arithmetic appear in children with learning disabilities of right cerebral origin". Experiments reveal that the thalamus on both sides was also involved.

Geary (26) asserts that human infants and "many other species are equipped with an innate set of fundamental quantitative competencies" at birth. The human infant brain is hard wired, as it were, to respond without formal training to increases and decreases in objects and events (up to four) in their immediate surroundings. He maintains that these abilities exist in infants of all races and unfold in a similar developmental pattern. Just as human infants are presumed to possess an innate capacity for language (85), mathematics, it is also claimed, has similar neurobiological foundations nurtured in enriching social environments (17). Such an arithmetic prototype resembles Chomsky's model of the universality of the basic elements of language commonly available to children regardless of race or culture.

Innate arithmetical abilities

Geary has argued that cognitive operations specific to arithmetic competencies are available to infants and young children just as they have access to rudimentary grammatical structures and rules. He refers to this capacity as "biologically primary abilities". These "abilities" comprise the capacity to estimate amounts of objects or events up to four without counting. The competence also includes an understanding of the concept of more and less without the assistance of the language of number words (a preverbal number system). It is argued that these innate preverbal skills form the foundations for dependable arithmetical abilities during formal schooling. Geary has declared that later "secondary" quantitative abilities in children are moulded by such social influences, as schooling and cultural customs.

Using the technique of visual gaze, Antell and Keating (3) examined the "perception of numerical invariance" in 40 healthy new born infants (21-144 hours mean 53 hours). They found that infants were capable of discriminating "among visual stimulus arrays consisting of sets of 2 versus 3 or 4 ver- sus 6 black dots" ... "but not for the larger sets (4-6 and 6-4)" In a carefully designed experiment they noted that neonates possessed a "natural domain of competence". Criterion measures used were total time of visual fixation at the stimulus display during habituation. The authors sought to control the likelihood of neonates responding to such redundant cues of the stimulus as background brightness and area and total target configuration.

However, the need for cautious acceptance of the neurophysiological explanations is warranted. More convincing research support is needed to rule out the possible effects of extraneous cues on the numeric competence of infants.

The authors caution that while infants respond to the novel features of a visual stimulus they do not necessarily integrate the information in a meaningful manner. Infants nevertheless possess rudimentary innate rule-making skills which enable them to extract information from their surroundings. These abilities may be similar to those employed throughout infant schooling. Cultural differences and contrasting patterns of academic instruction, however, influence the acquisition of any behavioural skill in children including arithmetic.

\section{Innate self-regulatory abilities}

Piaget's contribution to dyscalculia is universally acknowledged and enjoys a firm place in the literature. Piaget $(62,63)$ contended that children possess an innate capacity to organise their environment in quantitative ways. Children from a variety of cultural, socio-economic and ethnic backgrounds engage in bountiful commerce with their environment. These interactions inform them about the characteristics of the objects and events they encounter. This interaction develops their appreciation of informal arithmetic. Their abilities are further enhanced by regular interactions with adults. Parents and grown-ups spontaneously instruct young children about the properties of objects and such events as size, quantity ordinal and cardinal features of their environment. Children's informal arithmetic is further reinforced by the language of arithmetic, (heavy, light, more, less, far, near) attributes connoting quantity and relationships.

Children are active learners and employ their own unique method of shaping and forming concepts of number at an early stage. Vygotsky (82) for example maintained that young children are equipped with efficient, informal arithmetic systems. As they grow older, simpler methods yield to more efficient techniques influenced by maturation, instructional practices and socio-cultural factors. For a more complete discussion of the topic see Ginsburg (33).

Piaget's description of the four sequential stages of intellectual development throws further light on the topic. Piaget asserted that during the third stage of development (concrete operations) seven to eleven year old children display a firmer 
grasp of number and spatial reasoning as well as cause and effect. It is at this stage that arithmetical difficulties in children emerge and are recognised by teachers. Das, Kirby, and Jarman (18) offered a contrasting view, based upon Luria's (54) model of the three functional units of the brain which control arousal, coding and planning behaviour. According to Das and his associates, disruptions to any unit or units may lead to disturbances in learning, including disorders in arithmetical ability. Kirby and Ashman (49) in a factor analytic scrutiny of the arithmetic scores of 121 fifth grade pupils identified four types of planning factors in arithmetic operations. These comprised scanning, rehearsal, clustering and metacognition. They concluded that a child's visual scanning skills and verbal fluency were the best predictors of arithmetical achievement.

\section{Savant numerical ability}

A rare phenomenon seldom reported in the literature is that of the exceptional numerical abilities of some individuals with autism. Treffert (79) declared that there were less than 100 cases reported in the literature. The condition occurs six times more frequently in boys than girls.

The term Idiot Savant was coined by Langdon Down in 1887. The reference to idiot was later abandoned because of its offensive and unscientific connotations. These individuals possess the unusual ability to name the precise day of the week, a past, present or future date will occur. Their phenomenal capacity for "calendrical calculation" has puzzled psychologists and neurologists alike. The savant's numerical ability includes extraordinary skills in applying arithmetic principles to problem solving tasks (13). Gillberg and Rasmussen (32) claimed that the disorder was the result of damage to chromosome 7 .

\section{Associated medical disorders}

\section{Gerstmann's syndrome}

A number of other disorders are associated with number difficulties in young children. In the early 1920, success in finger localisation was positively correlated with written arithmetic. The inspiration for these studies was drawn from Gerstmann who drew attention to a syndrome of cortical disorder which he described as an inability to "recognise, indicate on request, name, or choose with open eyes, individual fingers either on their own hands or the hands of others." Gerstmann (31) suggested that a deficiency in number operations (acalculia) was associated with this disorder. Strauss and Werner (76), Werner and Carrison (84) and later Kinsbourne and Warrington (48) examined the possibility that a deficiency in the finger schema contributed to arithmetic difficulties in children who exhibited certain clinical abnormalities observed earlier by Gerstmann.

Other disorders

There are a variety of disorders associated with the dyscalculic phenomenon. Gross-Tsur et al. (38) found that children with neurological disorders often exhibited signs of DC. Children suffering from epilepsy (70) displayed particular difficulty in mastering arithmetic skills. Children with chromosomal, metabolic, attentional deficits and hyperactive disorders revealed singular weaknesses in the subject $(22,61,77)$.

Berry et al. (9) noted that children treated for phenylketonuria (PKU) presented difficulties in arithmetic. Girls with Turner's syndrome encountered specific difficulty with calculation suggestive of right parietal lobe involvement with possible links to chromosomal defects (59). It is further suggested that lesions or impaired development of the parietal, temporal and occipital cortex could be implicated in developmental dyscalculia.

Matthews and Folk (57) observed that in children with mental retardation, the conceptual and abstract concept of numbers is more susceptible to aphasic attacks, brain damage, cerebral dysfunction or debility than the recognition or ability to write a number on request.

\section{Psychological factors}

Associated problems in language, memory and attention

Arithmetic difficulties may be associated with delayed language development. Such children encounter problems in receptive and expressive abilities in number operations and verbal weaknesses in number counting. Gross-Tsur et al. (37) confirmed the presence of DC in a group 61 children between third and sixth grades with disordered language skills and weaknesses in arithmetic The author claims that the disorder arose from damage to cognate cortical sites in the brain.

The difficulty some children experience in memorising arithmetic facts and recalling arithmetic rules in, for example, addition and multiplication contribute to weaknesses in those areas. Geary (26) has claimed that such children differ from their normal peers in two ways. They are less adept in employing computational procedures to solve arithmetical problems and poorer at retrieving basic arithmetic facts from long-term semantic memory. He has further argued that their difficulty stems from a fundamental neurological weakness, and is possibly inherited and not occasioned by maturational lag. 
Children with attention span difficulties and a pronounced inability to persist with an activity for an appropriate length of time are liable to experience problems with arithmetic. Others easily distracted by extraneous stimuli have difficulty in distinguishing figure from background when inspecting an array of numbers. Some children described as distractible, hyperactive, hyperkinetic or stimulus-bound are unable to ignore the irrelevant stimuli they receive. When carrying out arithmetical operations they may have unusual difficulty in inhibiting their response to the competing stimuli of extraneous digits and symbols, and selectively focus on and attend to a limited field. Alternatively certain children become unnaturally attached to a single stimulus for inappropriate periods of time and 'perseverate'. After an operation is carried out, the child may persist with the same operation. For example, if the child is asked to add $4+4$, the child will with the next problem 3-1 answer 4 (repeating the operation of addition) or given the problem of $4 \times 4$ answer 8 .

\section{Associated sensory and motor disorders}

Disorders of sensory and motor development are known to affect visual, spatial and conceptual abilities, which are the basic building blocks for establishing number and calculation competence in children. A number of related disorders are implicated in arithmetical weaknesses in young children. These include weaknesses in visual perception and perceptual integration.

Hebb (42) noted that the capacity to recognise and discriminate simple forms and shapes is a gradual and complex process. Eye movements contribute constantly and essentially to perceptual integration. Piaget $(62,63)$ drew attention to the role of spatial ability in the development of the number concept in young children.

In their analysis of the causes of arithmetic difficulties in brain injured children, Strauss and Lehtinen (75) argued that weaknesses in perception contribute to poor arithmetic attainment in some cerebral palsied children. Those authors hypothesised that brain injured children are specially prone to arithmetical weaknesses because the brain injured individual lacks the ability possessed by the normal child to discover spontaneously the significant relationships of the number system.

Accepting the proposition that a perceptual scheme of visual spatial organisation is the basis of calculation, it is reasonable to anticipate that the child, in whom the ability to construct such a perceptual scheme has been disturbed, will be hindered in any activities which require its use. Strauss and Lehtinen developed the point that such children would have difficulty in forming number concepts because of disordered visual spatial organisation. "So intimate is the association between the perception of numerical relationships and the ability to reason and abstract, that the lack of the former has been considered diagnostic of deficiency in the latter".

Problems in eye movement could also contribute to difficulties in tracking the positions of numbers in the various columns of units, tens, hundreds etc. in addition tasks. Children with cerebral palsy show an abnormally high incidence of ocular motor defects, particularly strabismus, and may have singular weaknesses when skills are dependent upon movement under visual control. Children with alternating squints, severe nystagmus, tunnel vision, or defective visual scanning may face particular difficulties in accurately tracking the arrangements and positions of digits their during calculations of a set of numbers.

Neurological studies confirm that children exhibit singular weaknesses in spatially ordering and representing numerical information, e.g. aligning digits in correct columns, not confusing the horizontal and vertical rows of figures, reversing the position of digits and adding zeros appropriately.

Lewis et al. (52) revealed that children with SAD performed normally on auditory-perceptual tasks but poorly on visuospatial items. On the other hand children with combined arithmetic and reading difficulties perform poorly on visuo-spatial tasks but even more poorly on verbal- and auditory-perceptual tests. It is speculated that the disorders of these clinical subtypes arise from different neuro-psychological deficits.

Gaddes (24) observed that Luria was influenced by Piaget in his views about the development of number concepts in young children, despite drawing on his clinical work with adults who had sustained brain injuries. Luria apparently "recognised the visual, spatial and finally conceptual aspects of number processes in that order as the child grows" but maintained that the concept of number and arithmetical processes were dependent upon intact spatial ability.

Young children build up knowledge of objects in space by information from bodily movements transmitted along various sensory channels. It is reasonable to assume that if they are restricted in their activities by motor handicaps, particularly in early life, they could be deprived of a wide range of vital sensori-motor experiences. Many children with cerebral palsy are deprived of opportunities to physically explore their environment and several suffer an abnormal reduction of crucial preschool experiences. They have limited scope for manipulating various objects and toys, and have fewer opportunities to participate in normal play activities, for example building tower blocks, pulling and pushing equipment, thereby limiting their capacity for developing the appropriate symbols and concepts of number and simple mathematics.

Haskell (41) had noted that children with spastic diplegia were poorer at arithmetic than were children with athetosis or ataxia. He speculated that significant motor limitations in early life may lead to visuo-spatial disorders which contribute to poor arithmetic skills in this sub-type of cerebral palsy. 


\section{Associated emotional and behavioural disorders}

The emotional difficulties of underachieving children have been well reported in several studies $(8,10,12,23,44,55,60$, 78). Girls appear to be more anxious than boys (46). Thus, it is difficult to isolate the cultural determinants of such a result in these studies.

Shalev (71) noted the strong association between behavioural disorders and arithmetic difficulties in a number of studies. The authors indicated that in clinically referred cases the arithmetic difficulties were more enduring.

Studies by Hembree (44) and Ramirez \& Dockweiler (64) revealed that Hispanic and Native Americans pupils demonstrated much higher levels of anxiety compared with other ethnic groups, whereas African and Anglo-Saxon Americans appear to show no differences in anxious behaviour.

There is some evidence which supports the view that temperamental characteristics are associated with arithmetical difficulties in children and that anxious children read in order to escape into fantasy (55). It is conjectured that flight from arithmetic into books relieves anxieties or aids in the resolution of conflicts. Biggs (10) has in a literature review of studies traced a relationship between emotional factors and poor arithmetic attainment. Apparently, fear of arithmetic, particularly working with numbers, is apparent in some disturbed children. More recent studies $(4,66)$ have confirmed that anxiety and fear of tests affects the arithmetic performance of children. The physiological correlates of high arousal, it is suggested, leads to poor attention, carelessness, distractibility and "intrusive internal dialogue" as well as excessive concern about inaccurate and poor performance. According to Gaddes (24), arithmetic provokes greater fear in young children than does reading or other non-structured tasks. He suggested that this fear was generated by the strict demands of the rules of arithmetic which imposed uncompromising adherence to the structures and functions inherent in arithmetic operations.

Early and pre-school experiences

Arithmetic weaknesses in young children may be associated with a variety of factors other than the presence of neurological and cognitive disorders. Social cultural factors, for example, pre-school experiences, social and play opportunities also help in preparing young children to master the rudiments of counting and calculation. The socio-economic status, home background and motivation of parents are decisive influences for early success. The adult who assumes the task of teaching the concept of number, and familiarises the child with the language of counting and calculation, contributes substantially to the acquisition of arithmetic skills in young children.

Children's play is crucial for the acquisition of a range of skills, including arithmetic ability. Play supplies children with pleasurable activity and the opportunity to spontaneously discover the numerical characteristics and distinctive features of their surroundings.

Children of most cultures engage in a variety of arithmeticrelated play $(68,86)$. At a later stage, children develop more complex arithmetic skills as a result of formal schooling and instruction $(14,30)$.

Children with access to an abundance of informal arithmetic experiences in social and play situations are better prepared to benefit from formal instruction on entering primary school (19). The benefits that flow from family and home circumstances which promote arithmetical understanding through play cannot be too strongly emphasised. It is not always recognised that home influences affect the arithmetical readiness of children when they attend primary school for the first time. Those fortunate enough to find school an extension of home tend to maintain the initial advantage they had over those less well prepared. It should be emphasised though that consistent parental support at home rather than social class or income level is a determinant of children's arithmetic success and enjoyment. Negative attitudes towards school at home lead to poor motivation and lowered aspirations in children, for wealthy and poor alike.

Saxe, Guberman, and Gearhart (67) underscored the importance of early social interactions between parents and their children in the development of early numerical skills, the child's grasp of number, arithmetic vocabulary and other number related skills. It is through the conscious manipulation of such play materials as bricks, cubes and matching rods in various play situations that the foundations of mathematical concepts are firmly laid. These physical transactions furnish the child with a repertoire of informal knowledge and prepares the child for more formal instruction at school.

Piaget has asserted that young children are incapable of forming concepts of objects unless they have abundant opportunities to perform physical actions with them. While he stressed the importance of such engagements which help establish number concepts and number vocabulary, he warned against the notion that the materials in themselves impart concepts to children. He argued that it is the conscious manipulation of the materials which makes evident the significance of these operations.

\section{Culture and instructional practices}

\section{Cross cultural studies}

Varying instructional traditions and styles within and between countries influence the development of arithmetical skills in young children. The amount and quality of instruction children 
receive unquestionably influence their competence levels. Stevenson, Lee, and Stigler (73) reported that Japanese and Taiwanese children spent longer hours studying a subject than did their American counterparts. Even within American schools considerable variation in instructional time has been noted. Stevenson and Stigler (72) maintained that attainment in the subject was less highly valued by Americans compared with Japanese and Chinese educational authorities. It is further claimed that Japanese teachers emphasise and pupils respond appropriately to intense demands to learn mathematics.

Ginsburg, Posner, and Russell (34) in a cross cultural study of West African children and adults with American controls examined the counting performance in mental arithmetic problems administered verbally. The results indicated that young African and American children who received formal schooling employed a similar pattern for half the number of tasks and used a "regrouping" technique for the remaining problems. The method used was to divide numbers into more manageable units, e.g. $(37+28=30+20+7+8)$ to solve addition problems.

In the case of the unschooled African children, one third use number facts (recalled correct answers quickly without calculation) and a quarter employ counting and regrouping.

As the children grew older, both American- and Africanschooled children employed the mental algorithm (numbers were operated upon as digits and not as tens and hundreds) more frequently in contrast to the unschooled African children who did not use the system at all. American and schooled African children used regrouping methods very successfully, whereas unschooled African were far less accurate when they used regrouping methods.

With adults the effects of schooling showed a similar trend to that observed in the middle-aged (mean age 12.6 years) children. There was far less reliance upon regrouping in contrast to unschooled African adults.

The authors claimed that as the children grew older, counting was less favoured, whereas unschooled individuals rely on memorising facts as well as using regrouping strategies. However, unschooled adults become as equally proficient as their schooled counterparts and are not disadvantaged when not employing mental algorithms.

It is claimed that the African can access an informal cognitive skill which its own culture favours. It is further claimed that practice and drill in daily activities enables untaught African children to master simple number facts. A further result of the study was that among children who attended schools, there were no cultural differences between American and African children on such arithmetic tasks as mental addition.

Among adults there were no differences in accuracy between those who a) attended schools and b) the unschooled subjects. The researchers concluded that schooling has limited effects on mental addition tasks. It neither enhances problem- solving skills nor overcomes major weaknesses in cognitive learning.

Apparently European children, unlike their Asian counterparts, encounter difficulties in mastering the " base 10" system. Carrying and borrowing arithmetic tasks present problems for European children because of their lack of proficiency in the area.

The language of arithmetic further decreases understanding of tasks. For example, the introduction of words such as "more or less" confuses children and tends to increase error rates in computation.

Teaching styles and instruction

The importance of teaching efficient strategies to children for problem solving is critical for mastery of these skills in both primary and secondary pupils. "There is little reason to doubt that the dominant factor in successful teaching is and will always remain the teacher's skill in nourishing, and sometimes arousing, the child's curiosity and interest and in providing a rich and challenging environment in which the child can find his own unique way toward understanding, knowledge and skill" (16).

The crucial role of the teacher in aiding children form arithmetical concepts by providing appropriate experiences is well recognised (40). Teachers need to secure the interest of the child by making the learning situation attractive and relevant. It is necessary to create an environment that arouses curiosity, provides challenging experiences and encourages children to experiment. The skilled teacher is one who maintains rapport with the child and knows when to intervene and what experiences are relevant for acquiring specific skills. Piaget has postulated two invariant sequences of development through which the child must proceed in order to comprehend mathematical concepts. At the pre-operational stage the child is unable to understand even the simplest quantitative relationships, but makes judgements, using crude perceptual approximations. Next comes the intuitive stage, during which the child appreciates the idea of conservation intellectually, although it is difficult to equate it with the apparent inconsistency of its perceptions. It is not until the third or operational stage that the child is capable of appreciating logical numbers with real understanding. According to Piaget, it would be futile to teach a child the concept of number before it has grasped the idea of conservation. Teachers should enable children to understand concepts rather than develop rote skills and be aware of the sequences in which these concepts develop and unfold.

Geary (26) claims that before a child enters primary school it is armed with a rudimentary grasp of addition, subtraction ordination and cardination. However, the level of proficiency reached is dependent upon a variety of factors, not the least being the style and quality of instruction, the cultural traditions 
and the generational influences. He has argued that there is a degree of uniformity in the emergence and development of these secondary arithmetic skills in countries and across generations. Variations arise due to the contextual circumstances of industrialised and agricultural societies. Mastery of secondary skills is nevertheless highly dependent upon systematic and orderly instructional practices and drill. The importance of regular drill and extensive practice for skill acquisition cannot be sufficiently stressed (11). Practice, however, should be judiciously paced and designed to relegate the mechanical operations of calculation to a lower order cognitive activity at an automatic level (7).

Some children fail to master these skills due to the unfavourable attitudes of teachers towards the subject itself. Also, teachers who have an inadequate understanding of number concepts themselves fail to instruct children adequately. Glennon (35) observed that the average seventh grade pupil mastered a mere $12.5 \%$ of the understanding necessary for awareness of the computational processes taught in grades one through six. Lyon (56) emphasised that a childs weakness in mathematics was closely related to instructor competence and teaching skills. Teachers who taught concepts prematurely to children were found to have received faulty training as teachers. It is useful to trace early interest in this aspect of the topic.

Dutton (19) in a study of 55 elementary school teachers' understanding of arithmetic concepts noted that a substantial number relied on traditional methods and mechanical procedures learned during their own school days. Cheney (15) found that teachers' failure to present arithmetic meaningfully to students stemmed from their poor understanding of the subject. Several teachers experienced difficulties in comprehend- ing specific arithmetical concepts and many primary grade teachers were unable to teach concepts beyond the simplest stages. Lovell (53) confirmed that the acquisition of mathematical concepts by children is dependent upon the adequate understanding by the teacher and the climate of opinion in which the child is reared. Geary reporting on the work of Husén (45) and Stevenson, Chen, and Lee (73) claimed that mathematics education in the United States is one of the poorest in the industrialised countries.

School attendance

Absence from school of both normal and disabled children affects attainment in arithmetic more than in reading or in most school subjects. Schonell and Schonell (69), maintained that because arithmetic involves understanding of sequential steps and children progress by mastering each step in succession, irregular attenders who miss critical stages find it hard to keep up with their peers as they have little to base their next work upon.

Teachers need to be aware of the feeling of anxiety which repeated absence from arithmetic lessons may create in children. Haskell (41) found that the use of programmed instruction for children with cerebral palsy had obvious advantages when continuity of attendance during the term was interrupted by illness or accident.

Acknowledgements Thankes are due to Dr. J. Hollenweger for translating several articles from German into English. I am also grateful to Professor Mike Steer for his insightful comments on the draft of this chapter. 


\section{References}

1. American Psychiatric Association (1987) Diagnostic and Statistical Manual of Mental Disorders, Third Edition. Washington D.C.: American Psychiatric Association

2. American Psychiatric Association (1994) Diagnostic and Statistical Manual of Mental Disorders, Fourth Edition. Washington D.C.: American Psychiatric Association

3. Antell SE, Keating DP (1983) Perception of numerical invariance in neonates. Child Development 54: 695-701

4. Arkin RM, Detchon CS, Maruyama, GM (1982) Roles of attribution, affect and cognitive interference in test anxiety. Journal of Personality and Social Psychology 43: 1111-1124

5. Ashcroft MH, Yamashita TS, Aram DM (1992) Mathematics performance in left and right brain-lesioned children. Brain and Cognition 19: 208-252

6. Badian NA (1983) Dyscalculia and nonverbal disorders of learning. In: Myklebust HR (ed) Progress in Learning Disabilities 5: 235-264. New York: Grune \& Stratton

7. Bahrick HP (1993) Extending the life span of knowledge. In: Penner LA, Batsche GM, Knoff HM, NelsonDL (eds) The Challenge in Mathematics and Science Education: Psychology's Response. Washington DC: American Psychological Association

8. Barakat MK (1951) Factors underlying the mathematical abilities of grammar school pupils. British Journal of Educational Psychology 21: 239-240

9. Berry HK, O'Grady DJ, Perlmutter LJ, Botinger MK (1979) Intellectual Development and academic achievement of children treated for Phenylkotenuria. Developmental Medicine and Child Neurology 21: 311-320

10. Biggs JB (1959) Attitudes to arithmeticnumber anxiety. Education Research 1, 3: 6-21

11. Briars D, Siegler RS (1984) A featural analysis of preschoolers' counting knowledge. Developmental Psychology 22: 607-618

12. Burt C (1937) The Backward Child. London: London University Press

13. Cavanagh $K$ (2000) Studying savant ability. The Psychologist 13: 133-134

14. Ceci SJ (1991) How much does schooling influence general intelligence and its cognitive components? A reassessment of the evidence. Developmental Psychology 27: 703-722
15. Cheney AP (1961) Evaluation of elementary school teachers' understanding of basic arithmetic concepts. Unpublished Master of Arts Thesis. University of California Los Angeles

16. Chomsky N (1970) Phonology and reading. In: Levin H, Williams JP (eds) Basic Studies on Reading (pp 3-18). New York: Basic Books

17. Cobb P, Yackel E, Wood T (1992) A constructivist alternative to the representational view of mind in mathematics education. Journal for Research in Mathematics Education 23: 2-23

18. Das JP, Kirby JR, Jarman RF (1970) Simultaneous and successive cognitive processes. New York: Academic Press

19. Dutton WH (1961) University students comprehension of arithmetical concepts, Arithmetic Teacher 8: 60-64

20. Dutton WH (1964) Evaluating Pupils Understanding of Arithmetic. Englewood Cliffs, New Jersey: Prentice-Hall

21. Ericsson KA, Krampe RT, Tresch-Römer C (1993) The role of deliberate practice in the acquisition of expert performance. Psychological Review 100: 363-406

22. Faraone SV, Biederman J, Lehman BK, Spencer T, Norman D, Seidman LJ, Kraus I, Perrin J, Chen WJ, Tsuang MT (1993) Intellectual performance and school failure in children with attention deficit hyperactivity disorder and in their siblings. Journal of Abnormal Psychology 102: 616-623

23. Feldhusen JK, Klausmeir HJ (1962) Anxiety, intelligence and achievement in children of low, average and high intelligence. Child Development 33: 403-409

24. Gaddes WH (1985) Learning Disabilities and Brain Function: A Neuropsychological Approach. ( $2^{\text {nd }}$ edition) New York: Springer-Verlag

25. Geary DC (1990) A componential analysis of an early learning deficit in mathematics. Journal of Experimental Child Psychology 49: $363-383$

26. Geary DC (1993) Mathematical disabilities: cognitive, neuropsychological, and genetic components. Psychological Bulletin 114: 345-362

27. Geary DC (1994) Children's Mathematical Development: Research and Practical Applications. Washington D.C.: American Psychological Association

28. Geary DC (1995) Reflections of evolution and culture in children's cognition: implications for mathematical development and instruction. American Psychologist 50: 24-37
29. Geary DC, Fan L, Tow-Thomas CC (1992) Numerical cognition: loci of ability differences comparing children from china and the United States. Psychological Science 3: 180-185

30. Gelman R (1993) A rational-constructivist account of early learning about numbers and objects. In: Medlin DL (ed) The Psychology of Learning and Motivation. Advances in Research and Theory (Vol. 30, pp 61-96), San Diego: Academic Press

31. Gerstmann J (1924) Fingeragnosie: Eine umschriebene Störung der Orientierung am eigenen Körper. Wiener Klinische Wochenschrift 37: 1010-1012

32. Gillberg C, Rasmussen P (1994) Brief report: four case histories and a literature review of Williams disorder and autistic behavior. Journal of Autism and Developmental Disorders 24: 381-392

33. Ginsburg HP (1997) Mathematics learning disabilities: a view from developmental psychology. Journal of Learning Disabilities 30: 20-33

34. Ginsburg HP, Posner JK, Russell RL (1981) The development of mental addition as a function of schooling and culture. Journal of Cross-Cultural Psychology 12: 163-179

35. Glennon VJ (1948) A study of the growth and mastery of certain basic mathematical understanding on seven educational levels. Unpublished Doctoral Dissertation, Harvard University

36. Gordon N (1992) Children with developmental dyscalculia. Developmental Medicine and Child Neurology 34: 459-463

37. Gross-Tsur V, Manor O, Shalev RS (1997) Comorbidity in elementary school children with developmental language disorders. Annals of Neurology (abstract) 42: 525

38. Gross-Tsur V, Manor O, Shalev RS (1993) Developmental dyscalculia, gender and the brain. Archives of Disease in Childhood 68: 510-512

39. Gross-Tsur V, Manor O, Shalev RS (1996) Developmental dyscalculia: prevalence and demographic features. Developmental Medicine and Child Neurology 38: 25-33

40. Haskell SH, Barrett EK (1993) The Education of Children with Physical and Neurological Disabilities. $3^{\text {rd }}$ edition. London: Chapman \& Hall

41. Haskell SH (1973) Arithmetic Disabilities in Cerebral Palsied Children: Programmed Instruction. A Remedial Approach. Springfield: Charles C. Thomas 
42. Hebb DO (1949) The Organization of Behaviour. New York: Wiley

43. Hecaen H, Albert ML (1978) Human Neuropsychology. New York: Wiley

44. Hembree R (1990) The nature, effect and relief of mathematics anxiety. Journal for Research in Mathematics Education 21: 33-46

45. Husen T (1967) International Study of Achievements in Mathematics: A Comparison of Twelve Countries (Vols. 1, 2). New York: Wiley

46. Hyde JS, Fennema E, Ryan M, Frost L, Hopp C (1990) Gender comparison of mathematics attitudes and affect: a metaanalysis. Psychology of Women Quarterly 14: $229-324$

47. Hyrd GW, Semrud-Clikeman M (1989) Dyslexia and Brain Morphology. Psychological Bulletin 106: 447-482

48. Kinsbourne M, Warringto EK (1968) The developmental Gerstmann's syndrome. Archives of Neurology 8: 490-501

49. Kirby JR, Ashman AF (1984) Planning skills and mathematics achievement: implications regarding disability. Journal of Psychoeducational Assessment 2: 9-22

50. Klauer KJ (1992) Mathematik mehr leistungsschwache Mädchen, im Lesen und Rechtschreiben mehr leistungsschwache Jungen? Zeitschrift für Entwicklungspsychologie und Pädagogische Psychologie 26: $48-65$

51. Kosc L (1974) Developmental dyscalculia. Journal of Learning Disabilities 7: 46-59

52. Lewis C, Hitch, GJ, Walker P (1994) The prevalence of specific arithmetic difficulties and specific reading difficulties in 9 to 10 year-old boys and girls. Journal of Child Psychology and Psychiatry 35: 283-292

53. Lovell K (1961) The Growth of Basic Mathematical and Scientific Concepts in Children. London: University of London Press

54. Luria AR (1973) The Working Brain. Harmondsworth: Penguin Books

55. Lynn R (1957) Temperamental characteristics related to disparity of attainment in reading. British Journal of Educational Psychology 27: 62-67

56. Lyon GR (1996) State of research. In: Cramer S, Ellis W (eds) Learning Disabilities: Lifelong Issues. Baltimore: Brooks Publishing

57. Matthews CG Folk E (1964) Finger localisation, intelligence and arithmetic in mentally retarded subjects. American Journal of Mental Deficiency 69: 107-113

58. McCloskey M, Aliminosa D, Sokol SM (1991) Facts, rules and procedures in normal calculation: evidence from singlepatient studies of impaired arithmetic fact retrieval. Brain and Cognition 17: 154-203
59. Money J (1973) Turner's syndrome and parietal lobe functions. Cortex 9: 387-393

60. Newman CJ, Krug O (1964) Problems in learning arithmetic in emotionally disabled children. Journal of Child Psychiatry 3: 413-429

61. Pennington BF, Gilger JW, Pauls D, Smith SA, Smith SD, DeFries JC (1991) Evidence for major gene transmission of developmental dyslexia. Journal of the American Medical Association 266: 1527-1534

62. Piaget J (1952) The Child's Conception of Number. London: Routledge and Kegan Paul

63. Piaget J (1953) How children form mathematical concepts. Scientific American 189: 74-79

64. Ramirez OM, Dockweiler CJ (1987) Mathematics anxiety: a systematic review. In: Schwarzer R, van der Ploeg HM, Spielberger CD (eds) Advances in Test Anxiety Research (Vol. 5, pp 157-175), Berwyn, PA: Swets North America

65. Rourke BP, Finlayson MAJ (1978) Neuropsychological significance of variation in patterns of academic performance: verbal and visual-spatial abilities. Journal of Abnormal Child Psychology 6: 121-133

66. Sarason IG (1984) Stress, anxiety and cognitive interference; reactions to tests. Journal of Personality and Social Psychology 46: 929-938

67. Saxe GB, Guberman SR, Gearhart M (1987) Social processes in early number development. Monograph of the Society for Research in Child Development 52: 2 (Serial Number 216)

68. Saxe GB (1982) Culture and the development of numerical cognition: studies among the Oksapmin of Papua New Guinea. In: Brainerd CJ (ed) Children's Logical and Mathematical Cognition: Progress in Cognitive Development Research (pp 157-176), New York: Springer-Verlag

69. Schonell FJ, Schonell FE (1957) Diagnosis and Remedial Teaching in Arithmetic, Edinburgh: Oliver \& Boyd

70. Seidenberg M, Beck N, Geisser M, Giordani B, Sackellaves JC, Berent S, Dreifuss FE, Boll TJ (1986) Academic achievement of children with epilepsy. Epilepsia 27: 753-759

71. Shalev R (1998) Developmental dyscalculia. In: Perat MJ (ed) New Developments in Child Neurology. Bologna: Monduzzi Editore

72. Stevenson HL, Stigler J (1992) The Learning Gap: Why our Schools are Failing and What We Can Learn from Japanese and Chinese Education. New York: Summit

73. Stevenson HL, Lee SS, Stigler J (1986) The mathematics achievement of Chinese, Japanese, and American Children. Science 58: 693-699
74. Stevenson HW, Chen C, Lee SY (1993) Mathematics achievement of Chinese, Japanese and American children: ten years later. Science 259: 53-58

75. Strauss AA, Lehtinen LE (1947) Psychopathology and Education of the BrainInjured Child. New York: Grune and Stratton

76. Strauss AA, Werner H (1938) Deficiency in the finger schema in relation to arithmetic disability (finger agnosia and acalculia). American Journal of Orthopsychiatry 8: 719-725

77. Temple CM, Carrey RA (1993) Intellectual functioning of children with Turner syndrome: a comparison of behavioural phenotypes. Developmental Medicine and Child Neurology 35: 361-369

78. Tobias S (1978) Overcoming Math Anxiety. New York: Norton

79. Treffert DA (1998) The idiot savant: a review of the syndrome. American Journal of Psychiatry 145: 563-572

80. Von Aster M (1994) Developmental dyscalculia in children: review of the literature and clinical validation. Acta Paedopsychiatrica 56: 169-178

81. Von Aster MG, Deloche G, Dellatollas G, Meier M (1997) Zahlenverarbeitung und Rechnen bei Schulkindern der 2. und 3. Klassenstufe: Eine vergleichende Studie französischsprachiger und deutschsprachiger Kinder. Zeitschrift für Entwicklungspsychologie und Pädagogische Psychologie, XXIX, 2: 151-166

82. Vygotsky LS (1978) Mind in Society: The Development of Higher Psychological Processes (M Cole, Translation) MA: Harvard University Press (First published in 1930)

83. Warrington EK (1982) The Fractionation of arithmetic skills: a single case study. Quarterly Journal of Experimental Psychology 34A: 31-51

84. Werner H, Carrison D (1942) Measurement and development of the finger schema in mentally retarded children: relation of arithmetic achievement to performance on the finger schema test. Journal of Educational Psychology 33: 252-264

85. Witelson SF (1987) Neurobiological aspects of language. Child Development 58: 653-688

86. Zaslavsky C (1973) Africa counts: number and pattern in African culture. Boston, MA: Prindle, Weber, Schmidt 